\title{
On the Evaluation of the Function
}

\author{
$\phi(\lambda)=\frac{1}{2 \pi i} \int_{\sigma-i \infty}^{\sigma+i \infty} e^{u \ln u+\lambda u} d u$ \\ for Real Values of $\lambda^{*}$ \\ Wolfgang Börsch-Supan ${ }^{1}$
}

(June 26, 1961)

\begin{abstract}
The evaluation of the function mentioned in the title is done by deriving asymptotic formulas with error terms for the large values of $|\lambda|$. For values of $\lambda$ in the remaining finite interval, Simpson's rule is applied after suitable transformation of the path of integration and restriction to a finite portion of this path. A table of the function is given together with guesses of the errors due to the discretization and the finite integration limits.
\end{abstract}

\section{Introduction}

In his investigation of the energy loss of fast particles by ionization, Landau [1] ${ }^{2}$ uses the function mentioned in the title in order to describe the transformation of the spectral distribution of energy. He gives a short description of this function for its real arguments, including a plot and an asymptotic formula for the function itself and its integral with respect to $\lambda$.

For the numerical computation of the energy loss of a certain beam of particles in a certain medium, this function has to be tabulated and/or approximated to a certain accuracy. This requires asymptotic formulas and the corresponding error bounds for large values of $\lambda$, and, furthermore, numerical evaluation of the integral for a certain set of finite values after a suitable transformation of the path of integration.

The results are given in the following paragraphs.

\section{Transformation of Path of Integration}

As pointed out by Landau [1], the evaluation of the integral can be done either directly, by choosing a suitable value $\sigma$, or by transforming the path of integration. In the former case the best choice is a line of integration passing through the saddle point of the integrand. The derivative of the integrand vanishes for

$$
u=e^{-(1+\lambda)},
$$

so the best choice is

$$
\sigma=e^{-(1+\lambda)}
$$

The integral can be transformed into a real one by introducing $y=\operatorname{Im}\{u\}$ as a new independent variable and by combining the complex conjugate values of the integrand for complex conjugate values

*This work, was sponsored by the Naval Research Laboratory, order 173/6536/60. 1 Present address: Institut für Praktische Mathematik, Technische Hochschule Darmstadt, Darmstadt, Germany.

2 Figures in brackets indicate the literature references at the end of this paper. of $u$. Using (1) we obtain the integral representation

$$
\begin{array}{r}
\phi(\lambda)=\frac{e^{-\sigma}}{\pi} \int_{0}^{\infty} \exp \left\{\frac{\sigma}{2} \ln \left(1+\frac{y^{2}}{\sigma^{2}}\right)-y \arctan \frac{y}{\sigma}\right\} \\
\cdot \cos \left\{y\left[\frac{1}{2} \ln \left(1+\frac{y^{2}}{\sigma^{2}}\right)-1\right]+\sigma \arctan \frac{y}{\sigma}\right\} d y .
\end{array}
$$

For large positive values of $\lambda$ this vertical line in the complex plane passes near the singularity of the integrand at the origin. Furthermore, the phase angle of the integrand varies very fast. Therefore this path of integration is not suitable for numerical integration. As the integrand is fast decreasing in the left half-plane for these values of $\lambda$, we pull the path of integration from $\sigma \pm i \infty$ to the left to $-\infty$. But the singularity at the origin prevents a further movement of the middle part of the path of integration. Since we have a branch point at the origin, we put a branch cut along the negative real axis and transform the integral into an integral along this branch cut, from $-\infty$ to 0 on the lower side and from 0 to $-\infty$ on the upper side. The integrand differs by the difference of $2 \pi$ in the imaginary part of $\ln u$. So we get

$$
\begin{aligned}
\phi(\lambda) & =\frac{1}{2 \pi i} \int_{-\infty}^{0}\left(e^{-\pi i u}-e^{\pi i u}\right) e^{u \ln |u|+\lambda u} d u \\
& =\frac{1}{\pi} \int_{0}^{\infty} e^{-v(\ln v+\lambda)} \sin \pi v d v .
\end{aligned}
$$

The singularity at the origin is now multiplied by a factor of the order $v$ if compared with the original integrand. Furthermore, the integrand decreases the faster the larger $\lambda$ is.

It turns out that there is a large range in which both formulas are of practical use, but formula (2) is preferable for large negative $\lambda$, and formula (3) for large positive $\lambda$. At the same time, these formulae provide asymptotic formulas for $\phi(\lambda)$, given already by Landau, but without any remainder terms or error bounds. We extend Landau's considerations in order to get such error bounds. 


\section{Asymptotic Formula for Large Negative $\lambda$}

For large negative $\lambda$ the asymptotic behavior of $\phi(\lambda)$ is obtained by applying the saddle-point method to eq (2). The integrand can be written in the form

where

$$
\exp \left\{\sigma f\left(\frac{y}{\sigma}\right)\right\} \cos \left\{\sigma g\left(\frac{y}{\sigma}\right)\right\}
$$

$$
\begin{aligned}
& f(t)=\frac{1}{2} \ln \left(1+t^{2}\right)-t \arctan t, \\
& g(t)=t\left(\frac{1}{2} \ln \left(1+t^{2}\right)-1\right)+\arctan t .
\end{aligned}
$$

The following inequalities can be proved by elementary considerations which are too lengthy to be reproduced here in detail:

$$
\begin{aligned}
-\frac{t^{2}}{2} \leq f(t) \leq-\frac{t^{2}}{2}+\frac{t^{4}}{12} & \text { for } t \geq 0 \\
f(t) \leq-t\left(\frac{t_{0}}{2}-\frac{t_{0}^{3}}{12}\right) & \text { for } t \geq t_{0}>0 \\
0 \leq g(t) \leq \frac{t^{3}}{6} & \text { for } t \geq 0
\end{aligned}
$$

Replacing $f(t)$ by $-\frac{t^{2}}{2}$ and $g(t)$ by 0 yields the asymptotic expression

$$
\phi(\lambda) \sim \frac{e^{-\sigma}}{\pi} \int_{0}^{\infty} \exp \left(-\frac{y^{2}}{2 \sigma}\right) d y=\frac{\sqrt{\sigma} e^{-\sigma}}{\sqrt{2 \pi}}
$$

which was given by Landau.

Let us now look for a lower bound of $\phi(\lambda)$ or for a lower bound of the integrand. We replace $f(t)$ by $-t^{2} / 2, \cos \sigma g$ by $1-(\sigma g)^{2} / 2$ and then $g(t)$ by $t^{3} / 6$. Integration yields the lower bound

$$
\phi(\lambda) \geq \frac{\sqrt{\sigma} e^{-\sigma}}{\sqrt{2 \pi}}\left(1-\frac{5}{24 \sigma}\right) .
$$

It is a little more complicated to get an upper bound, since different inequalities are to be used for small $t$ and for large $t$. We replace $\cos \sigma g$ by 1 for all $t, f(t)$ by the upper bounds given by (4a) for $t \leq t_{0}$ and given by (4b) for $t \geq t_{0}$, respectively. The quantity $t_{0}$ is chosen later, depending upon $\sigma$. For the sake of closed integration, the expression exp $\left\{t^{4} / 12\right\}$ is replaced by the upper bound $1+(\exp$ $\left.\left\{t_{0}^{4} / 12\right\}-1\right) t^{4} / t_{0}^{4}$ for $t \leq t_{0}$. Now the integration can be carried out, provided to $t_{0}<\sqrt{6}$, and we obtain

$$
\begin{aligned}
& \phi(\lambda) \leq \frac{e^{-\sigma}}{\pi} \int_{0}^{\sigma t_{0}} \exp \left\{-\frac{y^{2}}{2 \sigma}\right\}\left(1+\left[e^{\sigma t_{0}^{4} / 12}-1\right] \frac{y^{4}}{\sigma^{4} t_{0}^{4}}\right) d y \\
&+\frac{\exp \left\{-\sigma t_{0}\left(\frac{t_{0}}{2}-\frac{t_{0}^{3}}{12}\right)\right\}}{\frac{t_{0}}{2}-\frac{t_{0}^{3}}{12}}
\end{aligned}
$$

Since the integrand of the remaining integral is positive for $y>\sigma t_{0}$ too, we replace $\sigma t_{0}$ by $\infty$ and obtain

$$
\phi(\lambda) \leq \frac{\sqrt{\sigma} e^{-\sigma}}{\sqrt{2 \pi}}\left(1+\frac{3\left[e^{\sigma t_{0}^{4} / 12}-1\right]}{\sigma^{2} t_{0}^{4}}+\sqrt{\frac{8}{\pi}} \frac{e^{\sigma t_{0}^{4} / 12} \cdot e^{-\sigma t_{0}^{2} / 2}}{\sqrt{\sigma} t_{0}\left(1-t_{0}^{2} / 6\right)}\right) .
$$

The value of $t_{0}$ should be chosen in such a way that the sum of the two last terms is a minimum. But the equation determining this minimum for general $\sigma$ is rather complicated. So we restrict ourselves to a rather crude discussion and to the case of large $\sigma$. The second term in parentheses is minimized for a fixed $\sigma$ by letting $\sigma t_{0}^{4} \rightarrow 0$. Then this term goes to $1 /(4 \sigma)$. But the third term goes to infinity then. Now let us put $\sigma t_{0}^{4}$ equal to some constant, say $12 \epsilon$ for convenience. Then our inequality has the form

$\phi(\lambda) \leq \frac{\sqrt{\sigma} e^{-\sigma}}{\sqrt{2 \pi}}\left(1+\frac{e^{\epsilon}-1}{\epsilon} \cdot \frac{1}{4 \sigma}+\frac{2}{\sqrt{\pi}} \frac{e^{\epsilon} \cdot e^{-\sqrt{3 \sigma \epsilon}}}{\sqrt[4]{3 \sigma \epsilon}\left(1-\sqrt{\frac{\epsilon}{3 \sigma}}\right)}\right)$,

valid for every $\lambda$ and for every positive $\epsilon<3 \sigma$. Apparently, for $\sigma \rightarrow \infty$ the last term goes much faster to zero than the middle term. So one can take $\epsilon$ small, as long as $\sigma \cdot \epsilon$ remains large. Then the first term approaches $1 /(4 \sigma)$.

\section{Asymptotic Formula for Large Positive $\lambda$}

The asymptotic behavior of $\phi(\lambda)$ for large positive $\lambda$ can be seen from (3). In a manner similar to Landau's, we transform the independent variable $\lambda$ and the integration variable $v$. The reason is that the interval where the integrand is essentially different from zero and contributes to the integral essentially should be independent of $\lambda$ for the new integration variable $t$. Such a transformation is given by

$$
\begin{aligned}
& \lambda=w+\ln w+B \\
& v=\frac{t}{w},
\end{aligned}
$$

where the new variables are $w$ and $t$, and $B$ is a constant chosen below. So we get

$$
\phi(\lambda)=\frac{1}{\pi w} \int_{0}^{\infty} e^{-t} e^{-t(\ln t+B) / w} \sin \frac{\pi t}{w} d t .
$$

Taylor expansion of the second exponential factor yields

$$
\begin{aligned}
\phi(\lambda) & =\frac{1}{w} \int_{0}^{\infty} e^{-t} \sin \frac{\pi t}{w} d t-\frac{1}{\pi w^{2}} \int_{0}^{\infty} e^{-t} t(\ln t+B) \sin \frac{\pi t}{w} d t \\
& +\frac{1}{2 \pi w^{3}} \int_{0}^{\infty} e^{-t} t^{2}(\ln t+B)^{2} \sin \frac{\pi t}{w} e^{-\frac{\theta(t, w) \cdot t(\ln t+B)}{w}} d t,
\end{aligned}
$$

where $0<\theta=\theta(t, w)<1$.

In order to obtain a remainder term of the order $w^{-4}$ we would like to choose $B$ in such a way that 
the second term becomes zero. But then $B$ would depend upon $w$. Now the same goal can be reached with a constant $B$ if one develops the sin-term in a Taylor series too:

$$
\sin \frac{\pi t}{w}=\frac{\pi t}{w}-\frac{1}{6}\left(\frac{\pi t}{w}\right)^{3} \cos \frac{\theta_{1} \pi t}{w}
$$

where $0<\theta_{1}=\theta_{1}(t, w)<1$. Now we demand only that the part of the second integral corresponding to the first term in the Taylor expansion of $\sin \pi t / w$ disappears, i.e., $B$ is determined by the equation

$$
\int_{0}^{\infty} e^{-t} t^{2}(\ln t+B) d t=\Gamma^{\prime}(3)+B \Gamma(3)=0,
$$

which leads to

$$
B=C-\frac{3}{2}=-0.922784335 \ldots
$$

where $C$ is the Eulerian constant, often also called $\gamma$ (see [2], especially eq (4) of paragraph 1.1 and (9) of paragraph 1.7). Then it follows that

$$
\begin{aligned}
& \phi(\lambda)=\frac{1}{w^{2}+\pi^{2}}+\frac{\pi^{2}}{6 w^{5}} \int_{0}^{\infty} e^{-t} t^{4}(\ln t+B) \cos \frac{\theta_{1} \pi t}{w} d t \\
& +\frac{1}{2 \pi w^{3}} \int_{0}^{\infty} e^{-t} t^{2}(\ln t+B)^{2} \sin \frac{\pi t}{w} e^{-\frac{\theta t(\ln t+B)}{w}} d t .
\end{aligned}
$$

In the first integral we replace the cos-term by the lower bound -1 for $t \leq e^{-B}$, and by the upper bound 1 for $t \geq e^{-B}$. The resulting integral can be written as

$$
\int_{0}^{\infty} e^{-t} t^{4}(\ln t+B) d t-2 \int_{0}^{e-B} e^{-t} t^{4}(\ln t+B) d t \leq 17 .
$$

The first term is evaluated in terms of the gamma function and its derivative. The second term is estimated by using $-(\ln t+B) \leq t^{-1}$ and by some elementary calculations. These arguments show that the modulus of the integral in the second term of $\left(7^{*}\right)$ is smaller than 17 .

The third term of $\left(7^{*}\right)$ can be treated in a similar way. We replace the sin-term by its bound $\pi t / w$ and the exp-term containing $\theta$ by its maximum value $\exp \left\{w^{-1} \exp (-B-1)\right\}$. The remaining integral can be expressed in terms of the gamma function and its first and second derivatives (see [2], .l.c.).

So finally we have the asymptotic formula

$$
\phi(\lambda)=\frac{1}{w^{2}+\pi^{2}}+R(w),
$$

where

$$
|R(w)| \leq \frac{\frac{\pi^{2}}{2}-\frac{15}{4}}{w^{4}} \exp \left\{\frac{0.926}{w}\right\}+\frac{17 \pi^{2}}{6 w^{5}} \equiv R_{1}(w) .
$$

and $w$ and $\lambda$ are connected by (7).
It is more convenient to have the right hand sides of (8) and (9) in terms of $\lambda$. Therefore one needs easily computable estimates of $w$ in terms of $\lambda$. The uncertainty of $w$ due to this estimation induces an uncertainty in the right hand side of (8), which in turn can be expressed by a corresponding change of $\lambda$ in the left hand side of (8). Therefore, in addition, we need an estimate of $\phi^{\prime}(\lambda)$. Using the value

$$
w_{1}=\lambda\left(1-\frac{\ln \lambda+B}{\lambda+1}\right)
$$

as an approximation for $w$, we obtain the inequalities

$$
\begin{aligned}
\frac{1}{w_{1}^{2}+\pi^{2}}- & R_{1}\left(w_{1}\right) \\
- & \frac{(\ln \lambda+B)^{2} \exp \left\{0.926 /\left(w_{1}-\ln w_{1}-B\right)\right\}}{\left(w_{1}-\ln w_{1}-B\right)^{3} w_{1}^{2}\left(1+\lambda^{-1}\right)^{3}} \\
& \quad \times\left(1+w_{1}^{-1}\right) \\
\leq & \phi(\lambda) \leq \frac{1}{w_{1}^{2}+\pi^{2}}+R_{1}\left(w_{1}\right) .
\end{aligned}
$$

They are valid provided $w_{1} \geq e^{-} \quad=2.52$.

\section{Comments on the Table}

The numerical evaluation of (2) and (3) for a given value of $\lambda$ requires the introduction of finite step lengths and of finite integration limits. As far as finite integration limits are concerned, the errors introduced by this approximation can be estimated rigorously. The errors due to the use of finite step lengths and Simpsun's rule are much more involved if expressed in terms of easily computable quantities. Repeated estimates would lead to very po or final estimates. Therefore, a mixed theoreticalexperimental way of guessing these errors was adopted. These guesses and some test runs with halved step lengths allow the assumption that the relative error of the values of $\phi(\lambda)$ as given in the table below do not exceed the amounts shown below. Together with the estimates of errors due to finite integration limits, one obtains

$$
\text { |relative error in table } \mid \leq\left\{\begin{array}{cl}
1.8 \times 10^{-4} & \text { if }-4 \leq \lambda \leq 2 \\
3.2 \times 10^{-4} & \text { if } 2 \leq \lambda \leq 10 \\
7 \times 10^{-4} & \text { if } 10 \leq \lambda
\end{array}\right.
$$

For values of $\lambda \leq-4$ the formulas (5), (6), and (1) give the value of $\phi(\lambda)$ with a relative error not exceeding 1.5 percent. For values of $\lambda \geq 100$ the formulas (11), (10), and (9) provide values of $\phi(\lambda)$ with a relative error not exceeding 0.02 percent.

The notation of the numbers is almost obvious: e.g., $1.25 \mathrm{E}-04$ means $1.25 \times 10^{-4}$. 


\begin{tabular}{|c|c|c|c|c|c|c|c|}
\hline$\lambda$ & $\phi(\lambda)$ & $\lambda$ & $\phi(\lambda)$ & $\lambda$ & $\phi(\lambda)$ & $\lambda$ & $\phi(\lambda)$ \\
\hline $\begin{array}{l}-4.000 \\
-3.975 \\
-3.950 \\
-3.925 \\
-3.900\end{array}$ & $\begin{array}{l}\text { 3. } 3900 \mathrm{E}-09 \\
\text { 5. } 4974 \mathrm{E}-09 \\
\text { 8. } 8066 \mathrm{E}-09 \\
\text { 1. } 3940 \mathrm{E}-08 \\
\text { 2. } 1811 \mathrm{E}-08\end{array}$ & $\begin{array}{l}-2.500 \\
-2.475 \\
-2.450 \\
-2.425 \\
-2.400\end{array}$ & $\begin{array}{l}\text { 9. } 6369 \mathrm{E}-03 \\
\text { 1. } 0633 \mathrm{E}-02 \\
\text { 1. } 1700 \mathrm{E}-02 \\
\text { 1. } 2840 \mathrm{E}-02 \\
\text { 1. } 4054 \mathrm{E}-02\end{array}$ & $\begin{array}{l}-1.000 \\
-0.975 \\
-0.950 \\
-0.925 \\
-0.900\end{array}$ & $\begin{array}{l}\text { 1. } 5140 \mathrm{E}-01 \\
\text { 1. } 5335 \mathrm{E}-01 \\
\text { 1. } 5523 \mathrm{E}-01 \\
\text { 1. } 5705 \mathrm{E}-01 \\
\text { 1. } 5880 \mathrm{E}-01\end{array}$ & $\begin{array}{l}0.500 \\
0.525 \\
0.550 \\
0.575 \\
0.600\end{array}$ & $\begin{array}{l}\text { 1. } 6524 \mathrm{E}-01 \\
\text { 1. } 6433 \mathrm{E}-01 \\
\text { 1. } 6341 \mathrm{E}-01 \\
\text { 1. } 6247 \mathrm{E}-01 \\
\text { 1. } 6152 \mathrm{E}-01\end{array}$ \\
\hline $\begin{array}{l}-3.875 \\
-3.850 \\
-3.825 \\
-3.800 \\
-3.775\end{array}$ & $\begin{array}{l}\text { 3. } 3740 \mathrm{E}-08 \\
\text { 5. } 1619 \mathrm{E}-08 \\
\text { 7. } 8123 \mathrm{E}-08 \\
\text { 1. } 1700 \mathrm{E}-07 \\
\text { 1. } 7342 \mathrm{E}-07\end{array}$ & $\begin{array}{l}-2.375 \\
-2.350 \\
-2.325 \\
-2.300 \\
-2.275\end{array}$ & $\begin{array}{l}\text { 1. } 5344 \mathrm{E}-02 \\
\text { 1. } 6712 \mathrm{E}-02 \\
\text { 1. } 8157 \mathrm{E}-02 \\
\text { 1. } 9682 \mathrm{E}-02 \\
\text { 2. } 1285 \mathrm{E}-02\end{array}$ & $\begin{array}{l}-0.875 \\
-0.850 \\
-0.825 \\
-0.800 \\
-0.775\end{array}$ & $\begin{array}{l}\text { 1. } 6048 \mathrm{E}-01 \\
\text { 1. } 6208 \mathrm{E}-01 \\
\text { 1. } 6362 \mathrm{E}-01 \\
\text { 1. } 6509 \mathrm{E}-01 \\
\text { 1. } 6649 \mathrm{E}-01\end{array}$ & $\begin{array}{l}0.625 \\
0.650 \\
0.675 \\
0.700 \\
0.725\end{array}$ & $\begin{array}{l}\text { 1. } 6056 \mathrm{E}-01 \\
\text { 1. } 5959 \mathrm{E}-01 \\
\text { 1. } 5861 \mathrm{E}-01 \\
\text { 1. } 5762 \mathrm{E}-01 \\
\text { 1. } 5661 \mathrm{E}-01\end{array}$ \\
\hline $\begin{array}{l}-3.750 \\
-3.725 \\
-3.700 \\
-3.675 \\
-3.650\end{array}$ & $\begin{array}{l}\text { 2. } 5449 \mathrm{E}-07 \\
\text { 3. } 6984 \mathrm{E}-07 \\
\text { 5. } 3235 \mathrm{E}-07 \\
\text { 7. } 5919 \mathrm{E}-07 \\
\text { 1. } 0729 \mathrm{E}-06\end{array}$ & $\begin{array}{l}\text {-2. } 250 \\
-2.225 \\
-2.200 \\
-2.175 \\
-2.150\end{array}$ & $\begin{array}{l}\text { 2. } 2968 \mathrm{E}-02 \\
\text { 2. } 4731 \mathrm{E}-02 \\
\text { 2. } 6571 \mathrm{E}-02 \\
\text { 2. } 8490 \mathrm{E}-02 \\
\text { 3. } 0486 \mathrm{E}-02\end{array}$ & $\begin{array}{l}-0.750 \\
-0.725 \\
-0.700 \\
-0.675 \\
-0.650\end{array}$ & $\begin{array}{l}\text { 1. } 6781 \mathrm{E}-01 \\
\text { 1. } 6907 \mathrm{E}-01 \\
\text { 1. } 7026 \mathrm{E}-01 \\
\text { 1. } 7138 \mathrm{E}-01 \\
\text { 1. } 7243 \mathrm{E}-01\end{array}$ & $\begin{array}{l}0.750 \\
0.775 \\
0.800 \\
0.825 \\
0.850\end{array}$ & $\begin{array}{l}\text { 1. } 5561 \mathrm{E}-01 \\
\text { 1. } 5459 \mathrm{E}-01 \\
\text { 1. } 5357 \mathrm{E}-01 \\
\text { 1. } 5254 \mathrm{E}-01 \\
\text { 1. } 5150 \mathrm{E}-01\end{array}$ \\
\hline $\begin{array}{l}-3.625 \\
-3.600 \\
-3.575 \\
-3.550 \\
-3.525\end{array}$ & $\begin{array}{l}\text { 1. } 5029 \mathrm{E}-06 \\
\text { 2. } 0872 \mathrm{E}-06 \\
\text { 2. } 873 \mathrm{E}-06 \\
\text { 3. } 9259 \mathrm{E}-06 \\
\text { 5. } 3195 \mathrm{E}-06\end{array}$ & $\begin{array}{l}-2.125 \\
-2.100 \\
-2.075 \\
-2.050 \\
-2.025\end{array}$ & $\begin{array}{l}\text { 3. } 2558 \mathrm{E}-02 \\
\text { 3. } 4703 \mathrm{E}-02 \\
\text { 3. } 6921 \mathrm{E}-02 \\
\text { 3. } 9209 \mathrm{E}-02 \\
\text { 4. } 1565 \mathrm{E}-02\end{array}$ & $\begin{array}{l}-0.625 \\
-0.600 \\
-0.575 \\
-0.550 \\
-0.525\end{array}$ & $\begin{array}{l}\text { 1. } 7341 \mathrm{E}-01 \\
\text { 1. } 7433 \mathrm{E}-01 \\
\text { 1. } 7518 \mathrm{E}-01 \\
\text { 1. } 7596 \mathrm{E}-01 \\
\text { 1. } 7668 \mathrm{E}-01\end{array}$ & $\begin{array}{l}0.875 \\
0.900 \\
0.925 \\
0.950 \\
0.975\end{array}$ & $\begin{array}{l}\text { 1. } 5046 \mathrm{E}-01 \\
\text { 1. } 4942 \mathrm{E}-01 \\
\text { 1. } 4837 \mathrm{E}-01 \\
\text { 1. } 4732 \mathrm{E}-01 \\
\text { 1. } 4627 \mathrm{E}-01\end{array}$ \\
\hline $\begin{array}{l}-3.500 \\
-3.475 \\
-3.450 \\
-3.425 \\
-3.400\end{array}$ & $\begin{array}{l}\text { 7. } 1518 \mathrm{E}-06 \\
\text { 9. } 5423 \mathrm{E}-06 \\
\text { 1. } 2638 \mathrm{E}-05 \\
\text { 1. } 6616 \mathrm{E}-05 \\
\text { 2. } 1694 \mathrm{E}-05\end{array}$ & $\begin{array}{l}-2.000 \\
-1.975 \\
-1.950 \\
-1.925 \\
-1.900\end{array}$ & $\begin{array}{l}\text { 4. } 3985 \mathrm{E}-02 \\
\text { 4. } 6468 \mathrm{E}-02 \\
\text { 4. } 9010 \mathrm{E}-02 \\
\text { 5. } 1608 \mathrm{E}-02 \\
\text { 5. } 4259 \mathrm{E}-02\end{array}$ & $\begin{array}{l}-0.500 \\
-0.475 \\
-0.450 \\
-0.425 \\
-0.400\end{array}$ & $\begin{array}{l}\text { 1. } 7734 \mathbf{E}-01 \\
\text { 1. } 7793 \mathbf{E}-01 \\
\text { 1. } 7846 \mathbf{E}-01 \\
\text { 1. } 7893 \mathbf{E}-01 \\
\text { 1. } 7934 \mathbf{E}-01\end{array}$ & $\begin{array}{l}\text { 1. } 000 \\
\text { 1. } 100 \\
\text { 1. } 200\end{array}$ & $\begin{array}{l}\text { 1. } 4521 \mathrm{E}-01 \\
\text { 1. } 4097 \mathrm{E}-01 \\
\text { 1. } 3673 \mathrm{E}-01\end{array}$ \\
\hline $\begin{array}{l}-3.375 \\
-3.350 \\
-3.325 \\
-3.300 \\
-3.275\end{array}$ & $\begin{array}{l}\text { 2. } 8128 \mathrm{E}-05 \\
\text { 3. } 6227 \mathrm{E}-05 \\
\text { 4. } 6353 \mathrm{E}-05 \\
\text { 5. } 8931 \mathrm{E}-05 \\
\text { 7. } 4458 \mathrm{E}-05\end{array}$ & $\begin{array}{l}-1.875 \\
-1.850 \\
-1.825 \\
-1.800 \\
-1.775\end{array}$ & $\begin{array}{l}\text { 5. } 6958 \mathrm{E}-02 \\
\text { 5. } 9703 \mathrm{E}-02 \\
\text { 6. } 2488 \mathrm{E}-02 \\
\text { 6. } 5311 \mathrm{E}-02 \\
\text { 6. } 8167 \mathrm{E}-02\end{array}$ & $\begin{array}{l}-0.375 \\
-0350 \\
-0.325 \\
-0.300 \\
-0.275\end{array}$ & $\begin{array}{l}\text { 1. } 7970 \mathrm{E}-01 \\
\text { 1. } 7999 \mathrm{E}-01 \\
\text { 1. } 8023 \mathrm{E}-01 \\
\text { 1. } 8042 \mathrm{E}-01 \\
\text { 1. } 8055 \mathrm{E}-01\end{array}$ & $\begin{array}{l}\text { 1. } 400 \\
\text { 1. } 500 \\
\text { 1. } 600 \\
\text { 1. } 700\end{array}$ & $\begin{array}{l}\text { 1. } 2834 \mathrm{E}-01 \\
\text { 1. } 2423 \mathrm{E}-01 \\
\text { 1. } 2018 \mathrm{E}-01 \\
\text { 1. } 1622 \mathrm{E}-01\end{array}$ \\
\hline $\begin{array}{l}-3.250 \\
-3.225 \\
-3.200 \\
-3.175 \\
-3.150\end{array}$ & $\begin{array}{l}\text { 9. } 3505 \mathrm{E}-05 \\
\text { 1. } 1673 \mathrm{E}-04 \\
\text { 1. } 4489 \mathrm{E}-04 \\
\text { 1. } 7882 \mathrm{E}-04 \\
\text { 2. } 1949 \mathrm{E}-04\end{array}$ & $\begin{array}{l}-1.750 \\
-1.725 \\
-1.700 \\
-1.675 \\
-1.650\end{array}$ & $\begin{array}{l}\text { 7. } 1052 \mathrm{E}-02 \\
\text { 7. } 3961 \mathrm{E}-02 \\
\text { 7. } 6891 \mathrm{E}-02 \\
\text { 7. } 9837 \mathrm{E}-02 \\
\text { 8. } 2795 \mathrm{E}-02\end{array}$ & $\begin{array}{l}-0.250 \\
-0.225 \\
-0.200 \\
-0.175 \\
-0.150\end{array}$ & $\begin{array}{l}\text { 1. } 8063 \mathrm{E}-01 \\
\text { 1. } 8066 \mathrm{E}-01 \\
\text { 1. } 8064 \mathrm{E}-01 \\
\text { 1. } 8057 \mathrm{E}-01 \\
\text { 1. } 8046 \mathrm{E}-01\end{array}$ & $\begin{array}{l}\text { 1. } 900 \\
\text { 1. } 900 \\
\text { 2. } 000 \\
\text { 2. } 100 \\
\text { 2. } 200\end{array}$ & $\begin{array}{l}\text { 1. } 0858 \mathrm{E}-01 \\
\text { 1. } 0492 \mathrm{E}-01 \\
\text { 1. } 0136 \mathrm{E}-01 \\
\text { 9. } 7911 \mathrm{E}-02\end{array}$ \\
\hline $\begin{array}{l}-3.125 \\
-3.100 \\
-3.075 \\
-3.050 \\
-3.025\end{array}$ & $\begin{array}{l}\text { 2. } 6797 \mathrm{E}-04 \\
\text { 3. } 2545 \mathrm{E}-04 \\
\text { 3. } 9326 \mathrm{E}-04 \\
\text { 4. } 7282 \mathrm{E}-04 \\
\text { 5. } 6574 \mathrm{E}-04\end{array}$ & $\begin{array}{l}-1.625 \\
-1.600 \\
-1.575 \\
-1.550 \\
-1.525\end{array}$ & $\begin{array}{l}\text { 8. } 5760 \mathrm{E}-02 \\
\text { 8. } 8729 \mathrm{E}-02 \\
\text { 9. } 1696 \mathrm{E}-02 \\
\text { 9. } 4658 \mathrm{E}-02 \\
9.7611 \mathrm{E}-02\end{array}$ & $\begin{array}{l}-0.125 \\
-0.100 \\
-0.075 \\
-0.050 \\
-0.025\end{array}$ & $\begin{array}{l}\text { 1. } 8030 \mathrm{E}-01 \\
\text { 1. } 8009 \mathrm{E}-01 \\
\text { 1. } 7984 \mathrm{E}-01 \\
\text { 1. } 7956 \mathrm{E}-01 \\
\text { 1. } 7923 \mathrm{E}-01\end{array}$ & $\begin{array}{l}\text { 2. } 300 \\
\text { 2. } 400 \\
\text { 2. } 500 \\
\text { 2. } 600 \\
\text { 2. } 700\end{array}$ & $\begin{array}{l}\text { 9. } 4573 \mathrm{E}-02 \\
\text { 9. } 1346 \mathrm{E}-02 \\
\text { 8. } 8230 \mathrm{E}-02 \\
\text { 8. } 5223 \mathrm{E}-02 \\
\text { 8. } 2324 \mathrm{E}-02\end{array}$ \\
\hline $\begin{array}{l}-3.000 \\
-2.975 \\
-2.950 \\
-2.925 \\
-2.900\end{array}$ & $\begin{array}{l}\text { 6. } 7372 \mathrm{E}-04 \\
\text { 7. } 9861 \mathrm{E}-04 \\
\text { 9. } 4241 \mathrm{E}-04 \\
\text { 1. } 1072 \mathrm{E}-03 \\
\text { 1. } 2953 \mathrm{E}-03\end{array}$ & $\begin{array}{l}-1.500 \\
-1.475 \\
-1.450 \\
-1.425 \\
-1.400\end{array}$ & $\begin{array}{l}\text { 1. } 0055 \mathrm{E}-01 \\
\text { 1. } 0347 \mathrm{E}-01 \\
\text { 1. } 0637 \mathrm{E}-01 \\
\text { 1. } 0925 \mathrm{E}-01 \\
\text { 1. } 1210 \mathrm{E}-01\end{array}$ & $\begin{array}{l}0.000 \\
0.025 \\
0.050 \\
0.075 \\
0.100\end{array}$ & $\begin{array}{l}\text { 1. } 7886 \mathrm{E}-01 \\
\text { 1. } 7845 \mathrm{E}-01 \\
\text { 1. } 7801 \mathrm{E}-01 \\
\text { 1. } 7754 \mathrm{E}-01 \\
\text { 1. } 7703 \mathrm{E}-01\end{array}$ & $\begin{array}{l}\text { 2. } 800 \\
\text { 2. } 900 \\
\text { 3. } 000 \\
\text { 3. } 100 \\
\text { 3. } 200\end{array}$ & $\begin{array}{l}\text { 7. } 9531 \mathrm{E}-02 \\
\text { 7. } 6842 \mathrm{E}-02 \\
\text { 7. } 4253 \mathrm{E}-02 \\
\text { 7. } 1763 \mathrm{E}-02 \\
\text { 6. } 9368 \mathrm{E}-02\end{array}$ \\
\hline $\begin{array}{l}-2.875 \\
-2.850 \\
-2.825 \\
-2.800 \\
-2.775\end{array}$ & $\begin{array}{l}\text { 1. } 5090 \mathrm{E}-03 \\
\text { 1. } 7509 \mathrm{E}-03 \\
\text { 2. } 0234 \mathrm{E}-03 \\
\text { 2. } 3293 \mathrm{E}-03 \\
\text { 2. } 6714 \mathrm{E}-03\end{array}$ & $\begin{array}{l}-1.375 \\
-1.350 \\
-1.325 \\
-1.300 \\
-1.275\end{array}$ & $\begin{array}{l}\text { 1. } 1491 \mathrm{E}-01 \\
\text { 1. } 1769 \mathrm{E}-01 \\
\text { 1. } 2043 \mathrm{E}-01 \\
\text { 1. } 2313 \mathrm{E}-01 \\
\text { 1. } 2579 \mathrm{E}-01\end{array}$ & $\begin{array}{l}0.125 \\
0.150 \\
0.175 \\
0.200 \\
0.225\end{array}$ & $\begin{array}{l}\text { 1. } 7648 \mathrm{E}-01 \\
\text { 1. } 7591 \mathrm{E}-01 \\
\text { 1. } 7530 \mathrm{E}-01 \\
\text { 1. } 7467 \mathrm{E}-01 \\
\text { 1. } 7401 \mathrm{E}-01\end{array}$ & $\begin{array}{l}\text { 3. } 300 \\
\text { 3. } 400 \\
\text { 3. } 500 \\
\text { 3. } 600 \\
\text { 3. } 700\end{array}$ & $\begin{array}{l}\text { 6. } 7066 \mathrm{E}-02 \\
\text { 6. } 4853 \mathrm{E}-02 \\
\text { 6. } 2725 \mathrm{E}-02 \\
\text { 6. } 0681 \mathrm{E}-02 \\
\text { 5. } 8717 \mathrm{E}-02\end{array}$ \\
\hline $\begin{array}{l}-2.750 \\
-2.725 \\
-2.700 \\
-2.675 \\
-2.650\end{array}$ & $\begin{array}{l}\text { 3. } 0524 \mathrm{E}-03 \\
\text { 3. } 4752 \mathrm{E}-03 \\
\text { 3. } 9428 \mathrm{E}-03 \\
\text { 4. } 4581 \mathrm{E}-03 \\
\text { 5. } 0239 \mathrm{E}-03\end{array}$ & $\begin{array}{l}-1.250 \\
-1.225 \\
-1.200 \\
-1.175 \\
-1.150\end{array}$ & $\begin{array}{l}\text { 1. } 2840 \mathrm{E}-01 \\
\text { 1. } 3096 \mathrm{E}-01 \\
\text { 1. } 3346 \mathrm{E}-01 \\
\text { 1. } 3591 \mathrm{E}-01 \\
\text { 1. } 3831 \mathrm{E}-01\end{array}$ & $\begin{array}{l}\text { 0. } 250 \\
0.275 \\
0.300 \\
0.325 \\
0.350\end{array}$ & $\begin{array}{l}\text { 1. } 7332 \mathrm{E}-01 \\
\text { 1. } 7261 \mathrm{E}-01 \\
\text { 1. } 7188 \mathrm{E}-01 \\
\text { 1. } 7112 \mathrm{E}-01 \\
\text { 1. } 7033 \mathrm{E}-01\end{array}$ & $\begin{array}{l}\text { 3. } 800 \\
\text { 3. } 900 \\
\text { 4. } 000 \\
\text { 4. } 100 \\
\text { 4. } 200\end{array}$ & $\begin{array}{l}\text { 5. } 6830 \mathrm{E}-02 \\
\text { 5. } 5016 \mathrm{E}-02 \\
\text { 5. } 3274 \mathrm{E}-02 \\
\text { 5. } 1599 \mathrm{E}-02 \\
\text { 4. } 9990 \mathrm{E}-02\end{array}$ \\
\hline $\begin{array}{l}-2.625 \\
-2.600 \\
-2.575 \\
-2.550 \\
-2.525\end{array}$ & $\begin{array}{l}\text { 5. } 6431 \mathrm{E}-03 \\
\text { 6. } 3186 \mathrm{E}-03 \\
\text { 7. } 0532 \mathrm{E}-03 \\
\text { 7. } 8494 \mathrm{E}-03 \\
\text { 8. } 7098 \mathrm{E}-03\end{array}$ & $\begin{array}{l}-1.125 \\
-1.100 \\
-1.075 \\
-1.050 \\
-1.025\end{array}$ & $\begin{array}{l}\text { 1. } 4065 \mathrm{E}-01 \\
\text { 1. } 4292 \mathrm{E}-01 \\
\text { 1. } 4514 \mathrm{E}-01 \\
\text { 1. } 4729 \mathrm{E}-01 \\
\text { 1. } 4937 \mathrm{E}-01\end{array}$ & $\begin{array}{l}0.375 \\
0.400 \\
0.425 \\
0.450 \\
0.475\end{array}$ & $\begin{array}{l}\text { 1. } 6953 \mathrm{E}-01 \\
\text { 1. } 6871 \mathrm{E}-01 \\
\text { 1. } 6787 \mathrm{E}-01 \\
\text { 1. } 6701 \mathrm{E}-01 \\
\text { 1. } 6613 \mathrm{E}-01\end{array}$ & $\begin{array}{l}\text { 4. } 300 \\
\text { 4. } 400 \\
\text { 4. } 500 \\
\text { 4. } 600 \\
\text { 4. } 700\end{array}$ & $\begin{array}{l}\text { 4. } 8444 \mathrm{E}-02 \\
\text { 4. } 6959 \mathrm{E}-02 \\
\text { 4. } 5530 \mathrm{E}-02 \\
\text { 4. } 4156 \mathrm{E}-02 \\
\text { 4. } 2836 \mathrm{E}-02\end{array}$ \\
\hline
\end{tabular}




\begin{tabular}{|c|c|c|c|c|c|c|c|}
\hline$\lambda$ & $\phi(\lambda)$ & $\lambda$ & $\phi(\lambda)$ & $\lambda$ & $\phi(\lambda)$ & $\lambda$ & $\phi(\lambda)$ \\
\hline $\begin{array}{l}\text { 4. } 800 \\
\text { 4. } 900 \\
\text { 5. } 000 \\
\text { 5. } 100 \\
\text { 5. } 200\end{array}$ & $\begin{array}{l}\text { 4. } 1566 \mathrm{E}-02 \\
\text { 4. } 0344 \mathrm{E}-02 \\
\text { 3. } 9168 \mathrm{E}-02 \\
\text { 3. } 8037 \mathrm{E}-02 \\
\text { 3. } 6948 \mathrm{E}-02\end{array}$ & $\begin{array}{l}10.800 \\
10.900 \\
11.000 \\
11.100 \\
11.200\end{array}$ & $\begin{array}{l}\text { 1. } 0337 \mathrm{E}-02 \\
\text { 1. } 0155 \mathrm{E}-02 \\
\text { 9. } 9777 \mathrm{E}-03 \\
\text { 9. } 8044 \mathrm{E}-03 \\
\text { 9. } 6354 \mathrm{E}-03\end{array}$ & $\begin{array}{l}\text { 16. } 800 \\
\text { 16. } 900 \\
\text { 17. } 000 \\
\text { 17. } 100 \\
\text { 17. } 200\end{array}$ & $\begin{array}{l}\text { 4. } 2927 \mathrm{E}-03 \\
\text { 4. } 2412 \mathrm{E}-03 \\
\text { 4. } 1905 \mathrm{E}-03 \\
\text { 4. } 1407 \mathrm{E}-03 \\
\text { 4. } 0918 \mathrm{E}-03\end{array}$ & $\begin{array}{l}\text { 25. } 200 \\
25.400 \\
25.600 \\
25.800 \\
26.000\end{array}$ & $\begin{array}{l}\text { 1. } 8640 \mathrm{E}-03 \\
\text { 1. } 8337 \mathrm{E}-03 \\
\text { 1. } 8042 \mathrm{E}-03 \\
\text { 1. } 7753 \mathrm{E}-03 \\
\text { 1. } 7471 \mathrm{E}-03\end{array}$ \\
\hline $\begin{array}{l}\text { 5. } 300 \\
5.400 \\
5.500 \\
5.600 \\
5.700\end{array}$ & $\begin{array}{l}\text { 3. } 5900 \mathrm{E}-02 \\
\text { 3. } 4891 \mathrm{E}-02 \\
\text { 3. } 3919 \mathrm{E}-02 \\
\text { 3. } 2983 \mathrm{E}-02 \\
\text { 3. } 2080 \mathrm{E}-02\end{array}$ & $\begin{array}{l}\text { 11. } 300 \\
11.400 \\
11.500 \\
11.600 \\
11.700\end{array}$ & $\begin{array}{l}\text { 9. } 4706 \mathrm{E}-03 \\
9.3097 \mathrm{E}-03 \\
\text { 9. } 1527 \mathrm{E}-03 \\
\text { 8. } 9995 \mathrm{E}-03 \\
\text { 8. } 8499 \mathrm{E}-03\end{array}$ & $\begin{array}{l}\text { 17. } 300 \\
17.400 \\
17.500 \\
17.600 \\
17.700\end{array}$ & $\begin{array}{l}\text { 4. } 0437 \mathrm{E}-03 \\
\text { 3. } 9965 \mathrm{E}-03 \\
\text { 3. } 9500 \mathrm{E}-03 \\
\text { 3. } 9043 \mathrm{E}-03 \\
\text { 3. } 8594 \mathrm{E}-03\end{array}$ & $\begin{array}{l}\text { 26. } 200 \\
\text { 26. } 400 \\
26.600 \\
26.800 \\
27.000\end{array}$ & $\begin{array}{l}\text { 1. } 7196 \mathrm{E}-03 \\
\text { 1. } 6927 \mathrm{E}-03 \\
\text { 1. } 6664 \mathrm{E}-03 \\
\text { 1. } 6407 \mathrm{E}-03 \\
\text { 1. } 6156 \mathrm{E}-03\end{array}$ \\
\hline $\begin{array}{l}\text { 5. } 800 \\
5.900 \\
6.000 \\
6.100 \\
6.200\end{array}$ & $\begin{array}{l}\text { 3. } 1210 \mathrm{E}-02 \\
\text { 3. } 0372 \mathrm{E}-02 \\
\text { 2. } 9563 \mathrm{E}-02 \\
\text { 2. } 8783 \mathrm{E}-02 \\
\text { 2. } 8031 \mathrm{E}-02\end{array}$ & $\begin{array}{l}\text { 11. } 800 \\
11.900 \\
\text { 12. } 000 \\
\text { 12. } 100 \\
\text { 12. } 200\end{array}$ & $\begin{array}{l}\text { 8. } 7038 \mathrm{E}-03 \\
\text { 8. } 5612 \mathrm{E}-03 \\
\text { 8. } 4219 \mathrm{E}-03 \\
\text { 8. } 2858 \mathrm{E}-03 \\
\text { 8. } 1528 \mathrm{E}-03\end{array}$ & $\begin{array}{l}\text { 17. } 800 \\
17.900 \\
18.000 \\
18.100 \\
18.200\end{array}$ & $\begin{array}{l}\text { 3. } 8152 \mathrm{E}-03 \\
\text { 3. } 7718 \mathrm{E}-03 \\
\text { 3. } 7291 \mathrm{E}-03 \\
\text { 3. } 6870 \mathrm{E}-03 \\
\text { 3. } 6457 \mathrm{E}-03\end{array}$ & $\begin{array}{l}\text { 27. } 200 \\
\text { 27. } 400 \\
\text { 27. } 600 \\
\text { 27. } 800 \\
\text { 28. } 000\end{array}$ & $\begin{array}{l}\text { 1. } 5910 \mathrm{E}-03 \\
\text { 1. } 5670 \mathrm{E}-03 \\
\text { 1. } 5436 \mathrm{E}-03 \\
\text { 1. } 5206 \mathrm{E}-03 \\
\text { 1. } 4982 \mathrm{E}-03\end{array}$ \\
\hline $\begin{array}{l}6.300 \\
6.400 \\
6.500 \\
6.600 \\
6.700\end{array}$ & $\begin{array}{l}\text { 2. } 7305 \mathrm{E}-02 \\
\text { 2. } 6604 \mathrm{E}-02 \\
\text { 2. } 5927 \mathrm{E}-02 \\
\text { 2. } 5273 \mathrm{E}-02 \\
\text { 2. } 4642 \mathrm{E}-02\end{array}$ & $\begin{array}{l}\text { 12. } 300 \\
\text { 12. } 400 \\
\text { 12. } 500 \\
\text { 12. } 600 \\
\text { 12. } 700\end{array}$ & $\begin{array}{l}\text { 8. } 0229 \mathrm{E}-03 \\
\text { 7. } 8959 \mathrm{E}-03 \\
\text { 7. } 7718 \mathrm{E}-03 \\
\text { 7. } 6505 \mathrm{E}-03 \\
\text { 7. } 5318 \mathrm{E}-03\end{array}$ & $\begin{array}{l}\text { 18. } 300 \\
\text { 18. } 400 \\
\text { 18. } 500 \\
\text { 18. } 600 \\
\text { 18. } 700\end{array}$ & $\begin{array}{l}\text { 3. } 6050 \mathrm{E}-03 \\
\text { 3. } 5650 \mathrm{E}-03 \\
\text { 3. } 5257 \mathrm{E}-03 \\
\text { 3. } 4870 \mathrm{E}-03 \\
\text { 3. } 4489 \mathrm{E}-03\end{array}$ & $\begin{array}{l}\text { 28. } 200 \\
28.400 \\
28.600 \\
28.800 \\
29.000\end{array}$ & $\begin{array}{l}\text { 1. } 4762 \mathrm{E}-03 \\
\text { 1. } 4547 \mathrm{E}-03 \\
\text { 1. } 4337 \mathrm{E}-03 \\
\text { 1. } 4131 \mathrm{E}-03 \\
\text { 1. } 3929 \mathrm{E}-03\end{array}$ \\
\hline $\begin{array}{l}\text { 6. } 800 \\
\text { 6. } 900 \\
\text { 7. } 000 \\
\text { 7. } 100 \\
\text { 7. } 200\end{array}$ & $\begin{array}{l}\text { 2. } 4031 \mathrm{E}-02 \\
\text { 2. } 3442 \mathrm{E}-02 \\
\text { 2. } 2872 \mathrm{E}-02 \\
\text { 2. } 2320 \mathrm{E}-02 \\
\text { 2. } 1787 \mathrm{E}-02\end{array}$ & $\begin{array}{l}\text { 12. } 800 \\
\text { 12. } 900 \\
\text { 13. } 000 \\
\text { 13. } 100 \\
\text { 13. } 200\end{array}$ & $\begin{array}{l}\text { 7. } 4158 \mathrm{E}-03 \\
\text { 7. } 3024 \mathrm{E}-03 \\
\text { 7. } 1914 \mathrm{E}-03 \\
\text { 7. } 0828 \mathrm{E}-03 \\
\text { 6. } 9766 \mathrm{E}-03\end{array}$ & $\begin{array}{l}\text { 18. } 800 \\
18.900 \\
\text { 19. } 000 \\
\text { 19. } 100 \\
\text { 19. } 200\end{array}$ & $\begin{array}{l}\text { 3. } 4114 \mathrm{E}-03 \\
\text { 3. } 3745 \mathrm{E}-03 \\
\text { 3. } 3381 \mathrm{E}-03 \\
\text { 3. } 3024 \mathrm{E}-03 \\
\text { 3. } 2672 \mathrm{E}-03\end{array}$ & $\begin{array}{l}\text { 29. } 200 \\
\text { 29. } 400 \\
29.600 \\
29.800 \\
30.000\end{array}$ & $\begin{array}{l}\text { 1. } 3732 \mathrm{E}-03 \\
\text { 1. } 3538 \mathrm{E}-03 \\
\text { 1. } 3349 \mathrm{E}-03 \\
\text { 1. } 3164 \mathrm{E}-03 \\
\text { 1. } 2982 \mathrm{E}-03\end{array}$ \\
\hline $\begin{array}{l}\text { 7. } 300 \\
\text { 7. } 400 \\
\text { 7. } 500 \\
\text { 7. } 600 \\
\text { 7. } 700\end{array}$ & $\begin{array}{l}\text { 2. } 1271 \mathrm{E}-02 \\
\text { 2. } 0772 \mathrm{E}-02 \\
\text { 2. } 0289 \mathrm{E}-02 \\
\text { 1. } 9821 \mathrm{E}-02 \\
\text { 1. } 9368 \mathrm{E}-02\end{array}$ & $\begin{array}{l}\text { 13. } 300 \\
\text { 13. } 400 \\
\text { 13. } 500 \\
\text { 13. } 600 \\
\text { 13. } 700\end{array}$ & $\begin{array}{l}\text { 6. } 8727 \mathrm{E}-03 \\
\text { 6. } 7710 \mathrm{E}-03 \\
\text { f. } 6714 \mathrm{E}-03 \\
\text { 6. } 5739 \mathrm{E}-03 \\
\text { 6. } 4785 \mathrm{E}-03\end{array}$ & $\begin{array}{l}19.300 \\
19.400 \\
19.500 \\
19.600 \\
19.700\end{array}$ & $\begin{array}{l}\text { 3. } 2325 \mathrm{E}-03 \\
\text { 3. } 1984 \mathrm{E}-03 \\
\text { 3. } 1648 \mathrm{E}-03 \\
\text { 3. } 1317 \mathrm{E}-03 \\
\text { 3. } 0991 \mathrm{E}-03\end{array}$ & $\begin{array}{l}30.200 \\
30.400 \\
30.600 \\
30.800 \\
31.000\end{array}$ & $\begin{array}{l}\text { 1. } 2804 \mathrm{E}-03 \\
\text { 1. } 2630 \mathrm{E}-03 \\
\text { 1. } 2459 \mathrm{E}-03 \\
\text { 1. } 2292 \mathrm{E}-03 \\
\text { 1. } 2127 \mathrm{E}-03\end{array}$ \\
\hline $\begin{array}{l}\text { 7. } 800 \\
\text { 7. } 900 \\
\text { 8. } 000 \\
\text { 8. } 100 \\
\text { 8. } 200\end{array}$ & $\begin{array}{l}\text { 1. } 8930 \mathrm{E}-02 \\
\text { 1. } 8504 \mathrm{E}-02 \\
\text { 1. } 8092 \mathrm{E}-02 \\
\text { 1. } 7693 \mathrm{E}-02 \\
\text { 1. } 7306 \mathrm{E}-02\end{array}$ & $\begin{array}{l}\text { 13. } 800 \\
\text { 13. } 900 \\
\text { 14. } 000 \\
\text { 14. } 100 \\
\text { 14. } 200\end{array}$ & $\begin{array}{l}\text { 6. } 3850 \mathrm{E}-03 \\
\text { 6. } 2935 \mathrm{E}-03 \\
\text { 6. } 2039 \mathrm{E}-03 \\
\text { 6. } 1161 \mathrm{E}-03 \\
\text { 6. } 0301 \mathrm{E}-03\end{array}$ & $\begin{array}{l}\text { 19. } 800 \\
19.900 \\
20.000 \\
20.200\end{array}$ & $\begin{array}{l}\text { 3. } 0671 \mathrm{E}-03 \\
\text { 3. } 0355 \mathrm{E}-03 \\
\text { 3. } 0043 \mathrm{E}-03\end{array}$ & $\begin{array}{l}31.200 \\
31.400 \\
31.600 \\
31.800 \\
32.000\end{array}$ & $\begin{array}{l}\text { 1. } 1966 \mathrm{E}-03 \\
\text { 1. } 1809 \mathrm{E}-03 \\
\text { 1. } 1654 \mathrm{E}-03 \\
\text { 1. } 1502 \mathrm{E}-03 \\
\text { 1. } 1353 \mathrm{E}-03\end{array}$ \\
\hline $\begin{array}{l}\text { 8. } 300 \\
\text { 8. } 400 \\
\text { 8. } 500 \\
\text { 8. } 600 \\
\text { 8. } 700\end{array}$ & $\begin{array}{l}\text { 1. } 6930 \mathrm{E}-02 \\
\text { 1. } 6566 \mathrm{E}-02 \\
\text { 1. } 6213 \mathrm{E}-02 \\
\text { 1. } 5870 \mathrm{E}-02 \\
\text { 1. } 5537 \mathrm{E}-02\end{array}$ & $\begin{array}{l}14.300 \\
14.400 \\
14.500 \\
14.600 \\
14.700\end{array}$ & $\begin{array}{l}\text { 5. } 9458 \mathrm{E}-03 \\
\text { 5. } 8632 \mathrm{E}-03 \\
\text { 5. } 7823 \mathrm{E}-03 \\
\text { 5. } 7029 \mathrm{E}-03 \\
\text { 5. } 6252 \mathrm{E}-03\end{array}$ & $\begin{array}{l}\text { 20. } 400 \\
20.600 \\
20.800 \\
21.000\end{array}$ & $\begin{array}{l}\text { 2. } 8844 \mathrm{E}-03 \\
\text { 2. } 8270 \mathrm{E}-03 \\
\text { 2. } 7713 \mathrm{E}-03 \\
\text { 2. } 7172 \mathrm{E}-03\end{array}$ & $\begin{array}{l}32.200 \\
32.400 \\
32.600 \\
32.800 \\
33.000\end{array}$ & $\begin{array}{l}\text { 1. } 1207 \mathrm{E}-03 \\
\text { 1. } 1064 \mathrm{E}-03 \\
\text { 1. } 0923 \mathrm{E}-03 \\
\text { 1. } 0786 \mathrm{E}-03 \\
1.0650 \mathrm{E}-03\end{array}$ \\
\hline $\begin{array}{l}\text { 8. } 800 \\
\text { 8. } 900 \\
9.000 \\
9.100 \\
9.200\end{array}$ & $\begin{array}{l}\text { 1. } 5214 \mathrm{E}-02 \\
\text { 1. } 4900 \mathrm{E}-02 \\
\text { 1. } 4595 \mathrm{E}-02 \\
\text { 1. } 4299 \mathrm{E}-02 \\
\text { 1. } 4011 \mathrm{E}-02\end{array}$ & $\begin{array}{l}\text { 14. } 800 \\
\text { 14. } 900 \\
\text { 15. } 000 \\
\text { 15. } 100 \\
\text { 15. } 200\end{array}$ & $\begin{array}{l}\text { 5. } 5489 \mathrm{E}-03 \\
\text { 5. } 4742 \mathrm{E}-03 \\
\text { 5. } 4009 \mathrm{E}-03 \\
\text { 5. } 3290 \mathrm{E}-03 \\
\text { 5. } 2584 \mathrm{E}-03\end{array}$ & $\begin{array}{l}\text { 21. } 200 \\
\text { 21. } 400 \\
\text { 21. } 600 \\
\text { 21. } 800 \\
\text { 22. } 000\end{array}$ & $\begin{array}{l}\text { 2. } 6646 \mathrm{E}-03 \\
\text { 2. } 6135 \mathrm{E}-03 \\
\text { 2. } 5639 \mathrm{E}-03 \\
\text { 2. } 5156 \mathrm{E}-03 \\
\text { 2. } 4686 \mathrm{E}-03\end{array}$ & $\begin{array}{l}\text { 33. } 200 \\
33.400 \\
33.600 \\
33.800 \\
34.000\end{array}$ & $\begin{array}{l}\text { 1. } 0517 \mathrm{E}-03 \\
\text { 1. } 0387 \mathrm{E}-03 \\
\text { 1. } 0259 \mathrm{E}-03 \\
\text { 1. } 0133 \mathrm{E}-03 \\
\text { 1. } 0010 \mathrm{E}-03\end{array}$ \\
\hline $\begin{array}{l}\text { 9. } 300 \\
9.400 \\
9.500 \\
9.600 \\
9.700\end{array}$ & $\begin{array}{l}\text { 1. } 3731 \mathrm{E}-02 \\
\text { 1. } 3459 \mathrm{E}-02 \\
\text { 1. } 3195 \mathrm{E}-02 \\
\text { 1. } 2938 \mathrm{E}-02 \\
\text { 1. } 2688 \mathrm{E}-02\end{array}$ & $\begin{array}{l}\text { 15. } 300 \\
15.400 \\
\text { 15. } 500 \\
\text { 15. } 600 \\
\text { 15. } 700\end{array}$ & $\begin{array}{l}\text { 5. } 1893 \mathrm{E}-03 \\
\text { 5. } 1214 \mathrm{E}-03 \\
\text { 5. } 0548 \mathrm{E}-03 \\
\text { 4. } 9895 \mathrm{E}-03 \\
\text { 4. } 9254 \mathrm{E}-03\end{array}$ & $\begin{array}{l}\text { 22. } 200 \\
\text { 22. } 400 \\
\text { 22. } 600 \\
\text { 22. } 800 \\
\text { 23. } 000\end{array}$ & $\begin{array}{l}\text { 2. } 4229 \mathrm{E}-03 \\
\text { 2. } 3784 \mathrm{E}-03 \\
\text { 2. } 3352 \mathrm{E}-03 \\
\text { 2. } 2930 \mathrm{E}-03 \\
\text { 2. } 2520 \mathrm{E}-03\end{array}$ & $\begin{array}{l}34.200 \\
34.400 \\
34.600 \\
34.800 \\
35.000\end{array}$ & $\begin{array}{l}\text { 9. } 8884 \mathrm{E}-04 \\
\text { 9. } 7693 \mathrm{E}-04 \\
9.6523 \mathrm{E}-04 \\
\text { 9. } 5374 \mathrm{E}-04 \\
\text { 9. } 4245 \mathrm{E}-04\end{array}$ \\
\hline $\begin{array}{r}9.800 \\
9.900 \\
10.000 \\
10.100 \\
10.200\end{array}$ & $\begin{array}{l}\text { 1. } 2445 \mathrm{E}-02 \\
\text { 1. } 2208 \mathrm{E}-02 \\
\text { 1. } 1977 \mathrm{E}-02 \\
\text { 1. } 1753 \mathrm{E}-02 \\
\text { 1. } 1535 \mathrm{E}-02\end{array}$ & $\begin{array}{l}\text { 15. } 800 \\
15.900 \\
16.000 \\
16.100 \\
16.200\end{array}$ & $\begin{array}{l}\text { 4. } 8625 \mathrm{E}-03 \\
\text { 4. } 8007 \mathrm{E}-03 \\
\text { 4. } 7401 \mathrm{E}-03 \\
\text { 4. } 6805 \mathrm{E}-03 \\
\text { 4. } 6221 \mathrm{E}-03\end{array}$ & $\begin{array}{l}\text { 23. } 200 \\
23.400 \\
23.600 \\
23.800 \\
24.000\end{array}$ & $\begin{array}{l}\text { 2. } 2120 \mathrm{E}-03 \\
\text { 2. } 1731 \mathrm{E}-03 \\
\text { 2. } 1352 \mathrm{E}-03 \\
\text { 2. } 0982 \mathrm{E}-03 \\
\text { 2. } 0622 \mathrm{E}-03\end{array}$ & $\begin{array}{l}\text { 35. } 200 \\
35.400 \\
35.600 \\
35.800 \\
\text { 36. } 000\end{array}$ & $\begin{array}{l}\text { 9. } 3136 \mathrm{E}-04 \\
\text { 9. } 2046 \mathrm{E}-04 \\
\text { 9. } 0974 \mathrm{E}-04 \\
\text { 8. } 9922 \mathrm{E}-04 \\
\text { 8. } 8887 \mathrm{E}-04\end{array}$ \\
\hline $\begin{array}{l}\text { 10. } 300 \\
10.400 \\
10.500 \\
10.600 \\
10.700\end{array}$ & $\begin{array}{l}\text { 1. } 1322 \mathrm{E}-02 \\
\text { 1. } 1115 \mathrm{E}-02 \\
\text { 1. } 0913 \mathrm{E}-02 \\
\text { 1. } 0716 \mathrm{E}-02 \\
\text { 1. } 0524 \mathrm{E}-02\end{array}$ & $\begin{array}{l}\text { 16. } 300 \\
16.400 \\
16.500 \\
16.600 \\
16.700\end{array}$ & $\begin{array}{l}\text { 4. } 5647 \mathrm{E}-03 \\
\text { 4. } 5084 \mathrm{E}-03 \\
\text { 4. } 4530 \mathrm{E}-03 \\
\text { 4. } 3986 \mathrm{E}-03 \\
\text { 4. } 3452 \mathrm{E}-03\end{array}$ & $\begin{array}{l}\text { 24. } 200 \\
\text { 24. } 400 \\
\text { 24. } 600 \\
\text { 24. } 800 \\
\text { 25. } 000\end{array}$ & $\begin{array}{l}\text { 2. } 0271 \mathrm{E}-03 \\
\text { 1. } 9928 \mathrm{E}-03 \\
\text { 1. } 9594 \mathrm{E}-03 \\
\text { 1. } 9269 \mathrm{E}-03 \\
\text { 1. } 8951 \mathrm{E}-03\end{array}$ & $\begin{array}{l}\text { 36. } 200 \\
36.400 \\
36.600 \\
36.800 \\
37.000\end{array}$ & $\begin{array}{l}\text { 8. } 7869 \mathrm{E}-04 \\
\text { 8. } 6869 \mathrm{E}-04 \\
\text { 8. } 5886 \mathrm{E}-04 \\
\text { 8. } 4919 \mathrm{E}-04 \\
\text { 8. } 3968 \mathrm{E}-04\end{array}$ \\
\hline
\end{tabular}


Table of the Function $\phi(\lambda)$-Continued

\begin{tabular}{|c|c|c|c|c|c|c|c|}
\hline$\lambda$ & $\phi(\lambda)$ & $\lambda$ & $\phi(\lambda)$ & $\lambda$ & $\phi(\lambda)$ & $\lambda$ & $\phi(\lambda)$ \\
\hline $\begin{array}{l}37.200 \\
37.400 \\
37.600 \\
37.800 \\
38.000\end{array}$ & $\begin{array}{l}\text { 8. } 3033 \mathrm{E}-04 \\
\text { 8. } 2113 \mathrm{E}-04 \\
\text { 8. } 1208 \mathrm{E}-04 \\
\text { 8. } 0318 \mathrm{E}-04 \\
\text { 7. } 9442 \mathrm{E}-04\end{array}$ & $\begin{array}{l}\text { 45. } 600 \\
45.800 \\
46.000 \\
46.200 \\
46.400\end{array}$ & $\begin{array}{l}\text { 5. } 4406 \mathrm{E}-04 \\
\text { 5. } 3914 \mathrm{E}-04 \\
\text { 5. } 3429 \mathrm{E}-04 \\
\text { 5. } 2951 \mathrm{E}-04 \\
\text { 5. } 2478 \mathrm{E}-04\end{array}$ & $\begin{array}{l}59.000 \\
59.500 \\
60.000 \\
60.500 \\
61.000\end{array}$ & $\begin{array}{l}\text { 3. } 1903 \mathrm{E}-04 \\
\text { 3. } 1351 \mathrm{E}-04 \\
\text { 3. } 0813 \mathrm{E}-04 \\
\text { 3. } 0289 \mathrm{E}-04 \\
\text { 2. } 9778 \mathrm{E}-04\end{array}$ & $\begin{array}{l}80.000 \\
80.500 \\
81.000 \\
81.500 \\
82.000\end{array}$ & $\begin{array}{l}\text { 1. } 7017 \mathrm{E}-04 \\
\text { 1. } 6800 \mathrm{E}-04 \\
\text { 1. } 6588 \mathrm{E}-04 \\
\text { 1. } 6379 \mathrm{E}-04 \\
\text { 1. } 6174 \mathrm{E}-04\end{array}$ \\
\hline $\begin{array}{l}\text { 38. } 200 \\
\text { 38. } 400 \\
38.600 \\
\text { 38. } 800 \\
\text { 39. } 000\end{array}$ & $\begin{array}{l}\text { 7. } 8581 \mathrm{E}-04 \\
\text { 7. } 7733 \mathrm{E}-04 \\
\text { 7. } 6898 \mathrm{E}-04 \\
\text { 7. } 6077 \mathrm{E}-04 \\
\text { 7. } 5269 \mathrm{E}-04\end{array}$ & $\begin{array}{l}\text { 46. } 600 \\
46.800 \\
47.000 \\
47.200 \\
47.400\end{array}$ & $\begin{array}{l}\text { 5. } 2012 \mathrm{E}-04 \\
\text { 5. } 1552 \mathrm{E}-04 \\
\text { 5. } 1098 \mathrm{E}-04 \\
\text { 5. } 0650 \mathrm{E}-04 \\
\text { 5. } 0208 \mathrm{E}-04\end{array}$ & $\begin{array}{l}61.500 \\
62.000 \\
62.500 \\
63.000 \\
63.500\end{array}$ & $\begin{array}{l}\text { 2. } 9280 \mathrm{E}-04 \\
\text { 2. } 8794 \mathrm{E}-04 \\
\text { 2. } 8320 \mathrm{E}-04 \\
\text { 2. } 7857 \mathrm{E}-04 \\
\text { 2. } 7406 \mathrm{E}-04\end{array}$ & $\begin{array}{l}82.500 \\
83.000 \\
83.500 \\
84.000 \\
84.500\end{array}$ & $\begin{array}{l}\text { 1. } 5973 \mathrm{E}-04 \\
1.5775 \mathrm{E}-04 \\
1.5581 \mathrm{E}-04 \\
1.5391 \mathrm{E}-04 \\
1.5204 \mathrm{E}-04\end{array}$ \\
\hline $\begin{array}{l}39.200 \\
39.400 \\
39.600 \\
39.800 \\
40.000\end{array}$ & $\begin{array}{l}\text { 7. } 4474 \mathrm{E}-04 \\
\text { 7. } 3691 \mathrm{E}-04 \\
\text { 7. } 2920 \mathrm{E}-04 \\
\text { 7. } 2161 \mathrm{E}-04 \\
\text { 7. } 1413 \mathrm{E}-04\end{array}$ & $\begin{array}{l}\text { 47. } 600 \\
47.800 \\
48.000 \\
48.200 \\
48.400\end{array}$ & $\begin{array}{l}\text { 4. } 9771 \mathrm{E}-04 \\
\text { 4. } 9340 \mathrm{E}-04 \\
\text { 4. } 8915 \mathrm{E}-04 \\
\text { 4. } 8495 \mathrm{E}-04 \\
\text { 4. } 8080 \mathrm{E}-04\end{array}$ & $\begin{array}{l}64.000 \\
64.500 \\
65.000 \\
65.500 \\
66.000\end{array}$ & $\begin{array}{l}\text { 2. } 6965 \mathrm{E}-04 \\
\text { 2. } 6535 \mathrm{E}-04 \\
\text { 2. } 6115 \mathrm{E}-04 \\
\text { 2. } 5705 \mathrm{E}-04 \\
\text { 2. } 5304 \mathrm{E}-04\end{array}$ & $\begin{array}{l}85.000 \\
85.500 \\
86.000 \\
86.500 \\
87.000\end{array}$ & $\begin{array}{l}\text { 1. } 5021 \mathrm{E}-04 \\
\text { 1. } 4841 \mathrm{E}-04 \\
\text { 1. } 4664 \mathrm{E}-04 \\
\text { 1. } 4490 \mathrm{E}-04 \\
\text { 1. } 4319 \mathrm{E}-04\end{array}$ \\
\hline $\begin{array}{l}40.200 \\
40.400 \\
40.600 \\
40.800 \\
41.000\end{array}$ & $\begin{array}{l}\text { 7. } 0677 \mathrm{E}-04 \\
6.9953 \mathrm{E}-04 \\
\text { 6. } 9239 \mathrm{E}-04 \\
\text { 6. } 8536 \mathrm{E}-04 \\
\text { 6. } 7844 \mathrm{E}-04\end{array}$ & $\begin{array}{l}\text { 48. } 600 \\
48.800 \\
49.000 \\
49.200 \\
49.400\end{array}$ & $\begin{array}{l}\text { 4. } 7671 \mathrm{E}-04 \\
\text { 4. } 7267 \mathrm{E}-04 \\
\text { 4. } 6868 \mathrm{E}-04 \\
\text { 4. } 6474 \mathrm{E}-04 \\
\text { 4. } 6084 \mathrm{E}-04\end{array}$ & $\begin{array}{l}66.500 \\
67.000 \\
67.500 \\
68.000 \\
68.500\end{array}$ & $\begin{array}{l}\text { 2. } 4913 \mathrm{E}-04 \\
\text { 2. } 4531 \mathrm{E}-04 \\
\text { 2. } 4157 \mathrm{E}-04 \\
\text { 2. } 3792 \mathrm{E}-04 \\
\text { 2. } 3435 \mathrm{E}-04\end{array}$ & $\begin{array}{l}87.500 \\
88.000 \\
88.500 \\
89.000 \\
89.500\end{array}$ & $\begin{array}{l}\text { 1. } 4151 \mathrm{E}-04 \\
\text { 1. } 3987 \mathrm{E}-04 \\
\text { 1. } 3825 \mathrm{E}-04 \\
\text { 1. } 3665 \mathrm{E}-04 \\
\text { 1. } 3509 \mathrm{E}-04\end{array}$ \\
\hline $\begin{array}{l}\text { 41. } 200 \\
41.400 \\
41.600 \\
41.800 \\
42.000\end{array}$ & $\begin{array}{l}\text { 6. } 7161 \mathrm{E}-04 \\
6.6489 \mathrm{E}-04 \\
6.5827 \mathrm{E}-04 \\
6.5175 \mathrm{E}-04 \\
6.4532 \mathrm{E}-04\end{array}$ & $\begin{array}{l}49.600 \\
49.800 \\
50.000\end{array}$ & $\begin{array}{l}\text { 4. } 5700 \mathrm{E}-04 \\
\text { 4. } 5320 \mathrm{E}-04 \\
\text { 4. } 4945 \mathrm{E}-04\end{array}$ & $\begin{array}{l}69.000 \\
69.500 \\
70.000 \\
70.500 \\
71.000\end{array}$ & $\begin{array}{l}\text { 2. } 3085 \mathrm{E}-04 \\
\text { 2. } 2744 \mathrm{E}-04 \\
\text { 2. } 2410 \mathrm{E}-04 \\
\text { 2. } 2083 \mathrm{E}-04 \\
\text { 2. } 1764 \mathrm{E}-04\end{array}$ & $\begin{array}{l}90.000 \\
90.500 \\
91.000 \\
91.500 \\
92.000\end{array}$ & $\begin{array}{l}\text { 1. } 3355 \mathrm{E}-04 \\
\text { 1. } 3204 \mathrm{E}-04 \\
\text { 1. } 3055 \mathrm{E}-04 \\
\text { 1. } 2909 \mathrm{E}-04 \\
\text { 1. } 2765 \mathrm{E}-04\end{array}$ \\
\hline $\begin{array}{l}42.200 \\
42.400 \\
42.600 \\
42.800 \\
43.000\end{array}$ & $\begin{array}{l}\text { 6. } 3899 \mathrm{E}-04 \\
\text { 6. } 3275 \mathrm{E}-04 \\
\text { 6. } 2660 \mathrm{E}-04 \\
\text { 6. } 2053 \mathrm{E}-04 \\
\text { 6. } 1456 \mathrm{E}-04\end{array}$ & $\begin{array}{l}50.500 \\
51.000 \\
51.500 \\
52.000 \\
52.500\end{array}$ & $\begin{array}{l}\text { 4. } 4028 \mathrm{E}-04 \\
\text { 4. } 3138 \mathrm{E}-04 \\
\text { 4. } 2275 \mathrm{E}-04 \\
\text { 4. } 1437 \mathrm{E}-04 \\
\text { 4. } 0623 \mathrm{E}-04\end{array}$ & $\begin{array}{l}71.500 \\
72.000 \\
72.500 \\
73.000 \\
73.500\end{array}$ & $\begin{array}{l}\text { 2. } 1451 \mathrm{E}-04 \\
\text { 2. } 1145 \mathrm{E}-04 \\
\text { 2. } 0845 \mathrm{E}-04 \\
\text { 2. } 0552 \mathrm{E}-04 \\
\text { 2. } 0264 \mathrm{E}-04\end{array}$ & $\begin{array}{l}\text { 92. } 500 \\
93.000 \\
93.500 \\
94.000 \\
94.500\end{array}$ & $\begin{array}{l}\text { 1. } 2624 \mathrm{E}-04 \\
\text { 1. } 2485 \mathrm{E}-04 \\
\text { 1. } 2348 \mathrm{E}-04 \\
\text { 1. } 2213 \mathrm{E}-04 \\
\text { 1. } 2081 \mathrm{E}-04\end{array}$ \\
\hline $\begin{array}{l}43.200 \\
43.400 \\
43.600 \\
43.800 \\
44.000\end{array}$ & $\begin{array}{l}\text { 6. } 0867 \mathrm{E}-04 \\
\text { 6. } 0286 \mathrm{E}-04 \\
\text { 5. } 9713 \mathrm{E}-04 \\
\text { 5. } 9149 \mathrm{E}-04 \\
\text { 5. } 8592 \mathrm{E}-04\end{array}$ & $\begin{array}{l}\text { 53. } 000 \\
53.500 \\
54.000 \\
54.500 \\
55.000\end{array}$ & $\begin{array}{l}\text { 3. } 9833 \mathrm{E}-04 \\
\text { 3. } 9066 \mathrm{E}-04 \\
\text { 3. } 8321 \mathrm{E}-04 \\
\text { 3. } 7596 \mathrm{E}-04 \\
\text { 3. } 6892 \mathrm{E}-04\end{array}$ & $\begin{array}{l}\text { 74. } 000 \\
74.500 \\
75.000 \\
75.500 \\
76.000\end{array}$ & $\begin{array}{l}\text { 1. } 9983 \mathrm{E}-04 \\
\text { 1. } 9708 \mathrm{E}-04 \\
\text { 1. } 9438 \mathrm{E}-04 \\
\text { 1. } 9173 \mathrm{E}-04 \\
\text { 1. } 8914 \mathrm{E}-04\end{array}$ & $\begin{array}{l}\text { 95. } 000 \\
95.500 \\
96.000 \\
96.500 \\
97.000\end{array}$ & $\begin{array}{l}\text { 1. } 1951 \mathrm{E}-04 \\
\text { 1. } 1823 \mathrm{E}-04 \\
\text { 1. } 1696 \mathrm{E}-04 \\
\text { 1. } 1572 \mathrm{E}-04 \\
\text { 1. } 1450 \mathrm{E}-04\end{array}$ \\
\hline $\begin{array}{l}\text { 44. } 200 \\
44.400 \\
44.600 \\
44.800 \\
45.000\end{array}$ & $\begin{array}{l}\text { 5. } 8043 \mathrm{E}-04 \\
\text { 5. } 7502 \mathrm{E}-04 \\
\text { 5. } 6968 \mathrm{E}-04 \\
\text { 5. } 6441 \mathrm{E}-04 \\
\text { 5. } 5922 \mathrm{E}-04\end{array}$ & $\begin{array}{l}55.500 \\
56.000 \\
56.500 \\
57.000 \\
57.500\end{array}$ & $\begin{array}{l}\text { 3. } 6207 \mathrm{E}-04 \\
\text { 3. } 5541 \mathrm{E}-04 \\
\text { 3. } 4893 \mathrm{E}-04 \\
\text { 3. } 4263 \mathrm{E}-04 \\
\text { 3. } 3649 \mathrm{E}-04\end{array}$ & $\begin{array}{l}\text { 76. } 500 \\
77.000 \\
77.500 \\
78.000 \\
78.500\end{array}$ & $\begin{array}{l}\text { 1. } 8660 \mathrm{E}-04 \\
\text { 1. } 8411 \mathrm{E}-04 \\
\text { 1. } 8168 \mathrm{E}-04 \\
\text { 1. } 7928 \mathrm{E}-04 \\
\text { 1. } 7694 \mathrm{E}-04\end{array}$ & $\begin{array}{l}\text { 97. } 500 \\
98.000 \\
98.500 \\
99.000 \\
99.500\end{array}$ & $\begin{array}{l}\text { 1. } 1330 \mathrm{E}-04 \\
\text { 1. } 1212 \mathrm{E}-04 \\
\text { 1. } 1095 \mathrm{E}-04 \\
\text { 1. } 0980 \mathrm{E}-04 \\
\text { 1. } 0867 \mathrm{E}-04\end{array}$ \\
\hline $\begin{array}{l}\text { 45. } 200 \\
45.400\end{array}$ & $\begin{array}{l}\text { 5. } 5410 \mathrm{E}-04 \\
\text { 5. } 4905 \mathrm{E}-04\end{array}$ & $\begin{array}{l}58.000 \\
58.500\end{array}$ & $\begin{array}{l}\text { 3. } 3052 \mathrm{E}-04 \\
\text { 3. } 2470 \mathrm{E}-04\end{array}$ & $\begin{array}{l}79.000 \\
79.500\end{array}$ & $\begin{array}{l}\text { 1. } 7464 \mathrm{E}-04 \\
\text { 1. } 7239 \mathrm{E}-04\end{array}$ & 100. 000 & 1. $0756 \mathrm{E}-04$ \\
\hline
\end{tabular}

The numerical computations were done on the National Bureau of Standards' 704 computer. The author acknowledges the help of Miss R. Zucker, who programed the computations.

(Paper 65B4-63)

\section{References}

[1] L. Landau, On the energy loss of fast particles by ionization, J. Physics 8 (1944), 201-205.

[2] A. Erdelyi, W. Magnus, F. Oberhettinger, and F. G. Tricomi, Higher transcendental functions, Vol. I, New York-Toronto-London, 1953.

[3] F. B. Hildebrand, Introduction to numerical analysis, New York-Toronto-London, 1956. 